\title{
Urologie Kolumne
}

\section{„Was ich schon immer mal sagen wollte ..."}

\author{
Liebe Kolleginnen und Kollegen, \\ jetzt wäre die Gelegenheit ... !! \\ Bitte lesen Sie weiter: \\ unsere wunderbare Uro-Kolumne, deren aktuelle Ausgabe Sie ge- \\ rade lesen, ist in den letzten 25 (!) Monaten regelmäßig aus der \\ (spitzen) Feder unseres geschätzten Kollegen Dr. Wolfgang Büh- \\ mann (Sylt) gekommen. Er hat es mit seiner - mündlich wie schrift- \\ lich - unübertroffenen Eloquenz, dem Mut zu „Sagen, was ist “ und \\ einer ordentlichen Prise „Pfeffer und Salz" geschafft, auch den Hart- \\ gesottenen unter uns je nach Thema und Gusto mal ein Schmun- \\ zeln, mal ein Grübeln, mal auch ein Zusammenzucken (was wir \\ Urologinnen und Urologen aushalten können) abzugewinnen und \\ damit ordentlich aufzurütteln. An dieser Stelle, lieber Wolfgang, da- \\ für ein ganz herzliches Dankeschön!
}

\begin{abstract}
Aber
Wie Sie, als aufmerksame Leserin oder Leser, schon bemerkt haben, hat an dieser Stelle in der letzten Ausgabe bereits der erste „Neuschreiberling", Dr. Holger Uhthoff, mit dieser Tradition gebrochen und über "Glieder in der Urologie" sinniert.
\end{abstract}

\section{Was ist passiert?}

Nein, Holger Uhthoff ist weder eine Urlaubsvertretung von Wolfgang Bühmann, noch ist derselbe des Schreibens müde geworden. Vielmehr hat sich nach dem Motto - „Nichts ist stetiger als der Wandel" im DGU-Vorstand die Überlegung breit gemacht, ob es nicht auch eine gute Idee wäre, das bisherige Format der Uro-Kolumne weiter zu entwickeln und damit auch für andere Autoren zu öffnen. Gesagt getan! Es wurde das Uro-KolumnenHerausgeberteam (Bühmann, Uhthoff, Wülfing) benannt und dieses freut sich nun, Ihnen in den folgenden Ausgaben (bis zum nächsten Wandel) die unterschiedlichsten Autorinnen und Autoren mit ihren ganz eigenen Themen und Gedanken präsentieren zu können.

Und wer sich von Ihnen schon immer mal schreiben wollte, hat nun die Gelegenheit, loszuwerden, was er immer schon mal sagen wollte über: (es folgen ein paar Ideen): meine urologische Lieblings-OP - den Klimawandel - die besten Urologie-Podcasts - eigene autobiographische Aspekte - Bürokratieabbau - urologische Innovationsideen - einen Lobgesang über bekannte oder nicht bekannte HeldInnen der Urologie - berufspolitische Betrachtungen - Gender-Wahnsinn - die besten Urlaubsziele für Urologinnen und Urologen - was auch immer: melden Sie sich gerne bei uns, dem Herausgeberteam (pressestelle@dgu.de), mit Ideen, Vorschlägen oder fertigen Manuskripten und tragen
Redaktion (V.i.S. d. P.)

Dr. Wolfgang Bühmann, Sylt

Dr. Holger Uhthoff, Speyer

Prof. Dr. Christian Wülfing, Hamburg

Schwarz-weiß trifft es selten, in der Regel braucht es eine differenzierte Meinung, gerne auch eine Prise Humor und manchmal muss der Daumen in die Wunde - auch in der Urologie. Deshalb spitzen hier verschiedene Autoren aus der Urologie den Stift und nehmen in dieser Rubrik regelmäßig aktuelle Themen ins Visier.

Sie zur qualitativen Vielfalt dieser Kolumne bei. Wir freuen uns auf Sie!

Ihre

\section{Wolfgang Bühmann \\ Holger Uhthoff \\ Christian Wülfing}

